\title{
New Variants of Newton's Method for Nonlinear Unconstrained Optimization Problems
}

\author{
V. KANWAR ${ }^{1}$, Kapil K. SHARMA ${ }^{2}$, Ramandeep BEHL ${ }^{2}$ \\ ${ }^{1}$ University Institute of Engineering and Technology, Panjab University, Chandigarh160 014, India \\ ${ }^{2}$ Department of Mathematics, Panjab University, Chandigarh160 014, India \\ Email:vmithil@yahoo.co.in,kapilks@pu.ac.in,ramanbehl87@yahoo.in
}

\begin{abstract}
In this paper, we propose new variants of Newton's method based on quadrature formula and power mean for solving nonlinear unconstrained optimization problems. It is proved that the order of convergence of the proposed family is three. Numerical comparisons are made to show the performance of the presented methods. Furthermore, numerical experiments demonstrate that the logarithmic mean Newton's method outperform the classical Newton's and other variants of Newton's method. MSC: $65 \mathrm{H} 05$.
\end{abstract}

Keywords: Unconstrained Optimization, Newton's Method, Order of Convergence, Power Means, Initial Guess

\section{Introduction}

The celebrated Newton's method

$$
x_{n+1}=x_{n}-\frac{f^{\prime}\left(x_{n}\right)}{f^{\prime \prime}\left(x_{n}\right)}
$$

used to approximate the optimum of a function is one of the most fundamental tools in computational mathematics, operation research, optimization and control theory. It has many applications in management science, industrial and financial research, chaos and fractals, dynamical systems, stability analysis, variational inequalities and even to equilibrium type problems. Its role in optimization theory can not be overestimated as the method is the basis for the most effective procedures in linear and nonlinear programming. For a more detailed survey, one can refer [1-4] and the references cited therein. The idea behind the Newton's method is to approximate the objective function locally by a quadratic function which agrees with the function at a point. The process can be repeated at the point that optimizes the approximate function. Recently, many new modified Newton-type methods and their variants are reported in the literature [5-8]. One of the reasons for discussing one dimensional optimization is that some of the iterative methods for higher dimensional problems involve steps of searching extrema along certain directions in $\mathfrak{R}^{n}$ [8]. Finding the step size, $\lambda_{n}$, along the direction vector $d_{n}$ involves solving the sub problem to minimize $f\left(x_{n+1}\right)=f\left(x_{n}+\lambda_{n} d_{n}\right)$, which is a one dimensional search problem in $\lambda_{n}$ [9]. Hence the one dimensional methods are most indispensable and the efficiency of any method partly depends on them [10].

The purpose of this work is to provide some alternative derivations through power mean and to revisit some well-known methods for solving nonlinear unconstrained optimization problems.

\section{Review of Definition of Various Means}

For a given finite real number $\alpha$, the $\alpha$ th-power mean $m_{\alpha}$ of positive scalars $a$ and $b$, is defined as follows [11]

$$
m_{\alpha}=\left(\frac{a^{\alpha}+b^{\alpha}}{2}\right)^{\frac{1}{\alpha}}
$$

It is easy to see that

$$
\begin{aligned}
& \text { For } \alpha=-1, \quad m_{-1} \text { (Harmonic mean) }=\frac{2 a b}{a+b}, \\
& \text { For } \alpha=\frac{1}{2}, \quad m_{\frac{1}{2}}=\left\{\frac{\sqrt{a}+\sqrt{b}}{2}\right\}^{2}, \\
& \text { For } \alpha=1, \quad m_{1} \text { (Arithmetic mean) }=\frac{a+b}{2} . \\
& \text { For } \alpha \rightarrow 0, \text { we have } \lim _{\alpha \rightarrow 0} m_{\alpha}=\sqrt{a b},
\end{aligned}
$$

which is the so-called geometric mean of $a, b$ and may be denoted by $m_{g}$. 
For given positive scalars $a$ and $b$, some other well-known means are defined as

$$
\begin{aligned}
& N \text { (Heronian mean) }=\frac{a+\sqrt{a b}+b}{3}, \\
& C \text { (Contra-harmonic mean) }=\frac{a^{2}+b^{2}}{a+b}, \\
& T \text { (Centroidal mean) }=\frac{2\left(a^{2}+a b+b^{2}\right)}{3(a+b)},
\end{aligned}
$$

and

$$
L \quad(\text { Logarithmic mean })=\frac{a-b}{\log (a)-\log (b)} .
$$

\section{Variants of Newton's Method for Nonlinear Equations}

Recently, some modified Newton's method with cubic convergence have been developed by considering different quadrature formula for computing integral, arising in the Newton's theorem [12]

$$
f(x)=f\left(x_{n}\right)+\int_{x_{n}}^{x} f^{\prime}(t) d t
$$

Weerakoon and Fernando [13] re-derived the classical Newton's method by approximating the indefinite integral in (11) by rectangular rule. Suppose that $x=x_{n+1}$ is the root of the equation $f(x)=0$, we then put the left side $f\left(x_{n+1}\right)=0$ in the identity (11) and approximate the integral by the rectangular rule according to which

$$
\int_{x_{n}}^{x_{n+1}} f^{\prime}(t) d t \cong\left(x_{n+1}-x_{n}\right) f^{\prime}\left(x_{n}\right)
$$

Therefore, from (11) and (12), they obtain the wellknown Newton's method. Weerakoon and Fernando [13] further used trapezoidal approximation to the definite integral according to which

$$
\int_{x_{n}}^{x_{n+1}} f^{\prime}(t) d t \cong \frac{1}{2}\left(x_{n+1}-x_{n}\right)\left\{f^{\prime}\left(x_{n}\right)+f^{\prime}\left(x_{n+1}\right)\right\}
$$

to obtain modified Newton's method given by

$$
x_{n+1}=x_{n}-\frac{2 f\left(x_{n}\right)}{f^{\prime}\left(x_{n}\right)+f^{\prime}\left(x_{n+1}^{\bullet}\right)}
$$

where

$$
x_{n+1}^{\bullet}=x_{n}-\frac{f\left(x_{n}\right)}{f^{\prime}\left(x_{n}\right)}
$$

is the Newton's iterate. In contrast to classical Newton's method, this method uses the arithmetic mean of $f^{\prime}\left(x_{n}\right)$ and $f^{\prime}\left(x_{n+1}^{*}\right)$, instead of $f^{\prime}\left(x_{n}\right)$. Therefore, it is called arithmetic mean Newton's method [13]. By using different approximations to the indefinite integral in the Newton's theorem (11), different iterative formulas can be obtained for solving nonlinear equations [14].

\section{Variants of Newton's Method for Unconstrained Optimization Problems}

Now we shall extend this idea for the case of unconstrained optimization problems. Suppose that the function $f(x)$ is a sufficiently differentiable function. Let $\beta \in \mathfrak{R}$ is an extremum point of $f(x)$, then $x=\beta$ is a root of

$$
f^{\prime}(x)=0
$$

Extending Newton's theorem (11), we have

$$
f^{\prime}(x)=f^{\prime}\left(x_{n}\right)+\int_{x_{n}}^{x} f^{\prime \prime}(t) d t
$$

Approximating the indefinite integral in (17) by the rectangular rule according to which

$$
\int_{x_{n}}^{x_{n+1}} f^{\prime \prime}(t) d t \cong\left(x_{n+1}-x_{n}\right) f^{\prime \prime}\left(x_{n}\right)
$$

and using $f^{\prime}\left(x_{n+1}\right)=0$, we get

$$
x_{n+1} \equiv x_{n+1}^{N}=x_{n}-\frac{f^{\prime}\left(x_{n}\right)}{f^{\prime \prime}\left(x_{n}\right)}, f^{\prime \prime}\left(x_{n}\right) \neq 0 .
$$

This is a well-known quadratically convergent Newton's method for unconstrained optimization problems.

Approximating the integral in (17) by the trapezoidal approximation

$$
\int_{x_{n}}^{x_{n+1}} f^{\prime \prime}(t) d t \cong \frac{1}{2}\left(x_{n+1}-x_{n}\right)\left\{f^{\prime \prime}\left(x_{n}\right)+f^{\prime \prime}\left(x_{n+1}\right)\right\}
$$

in combination with the approximation

$$
f^{\prime \prime}\left(x_{n+1}\right) \approx f^{\prime \prime}\left\{x_{n}-\frac{f^{\prime}\left(x_{n}\right)}{f^{\prime \prime}\left(x_{n}\right)}\right\} \equiv f^{\prime \prime}\left(x_{n+1}^{N}\right) \text { and } f^{\prime}\left(x_{n+1}\right)=0 \text {, }
$$

we get the following arithmetic mean Newton's method given by

$$
x_{n+1}=x_{n}-\frac{2 f^{\prime}\left(x_{n}\right)}{f^{\prime \prime}\left(x_{n}\right)+f^{\prime \prime}\left(x_{n+1}^{N}\right)}
$$

for unconstrained optimization problems. This formula is also derived independently by Kahya [5].

If we use the midpoint rule of integration in (20) (Gaussian-Legendre formula with one knot) [15], then we obtain a new formula given by 


$$
x_{n+1}=x_{n}-\frac{f^{\prime}\left(x_{n}\right)}{f^{\prime \prime}\left(\frac{x_{n}+x_{n+1}^{N}}{2}\right)}
$$

This formula may be called the midpoint Newton's formula for unconstrained optimization problems. Now for generalization, approximating the functions in correction factor in (21) with a power means of $a=f^{\prime \prime}\left(x_{n}\right)$ and $b=f^{\prime \prime}\left(x_{n+1}^{N}\right)$, we have

$$
x_{n+1}=x_{n}-\frac{f^{\prime}\left(x_{n}\right)}{\operatorname{sign}\left\{f^{\prime \prime}\left(x_{0}\right)\right\}\left[\frac{f^{\prime \prime \alpha}\left(x_{n}\right)+f^{\prime \prime \alpha}\left(x_{n+1}^{N}\right)}{2}\right]^{\frac{1}{\alpha}}}
$$

This family may be called the $\alpha$ th-power mean iterative family of Newton's method for unconstrained optimization problems.

Special cases:

It is interesting to note that for different specific values of $\alpha$, various new methods can be deduced from For- mula (23) as follows:

1) For $\alpha=1$ (arithmetic mean), Formula (23) corresponds to cubically convergent arithmetic mean Newton's method

$$
x_{n+1}=x_{n}-\frac{2 f^{\prime}\left(x_{n}\right)}{f^{\prime \prime}\left(x_{n}\right)+f^{\prime \prime}\left(x_{n+1}^{N}\right)}
$$

2) For $\alpha=-1$ (harmonic mean), Formula (23) corresponds to a cubically convergent harmonic mean Newton's method

$$
x_{n+1}=x_{n}-\frac{f^{\prime}\left(x_{n}\right)}{2}\left[\frac{1}{f^{\prime \prime}\left(x_{n}\right)}+\frac{1}{f^{\prime \prime}\left(x_{n+1}^{N}\right)}\right]
$$

3) For $\alpha \rightarrow 0$ (geometric mean), Formula (23) corresponds to a new cubically convergent geometric mean Newton's method

$$
x_{n+1}=x_{n}-\frac{f^{\prime}\left(x_{n}\right)}{\operatorname{sign}\left\{f^{\prime \prime}\left(x_{0}\right)\right\} \sqrt{\left\{f^{\prime \prime}\left(x_{n}\right) f^{\prime \prime}\left(x_{n+1}^{N}\right)\right\}}}
$$

4) For $\alpha=\frac{1}{2}$, Formula (23) corresponds to a new cubically convergent method

$$
x_{n+1}=x_{n}-\frac{4 f^{\prime}\left(x_{n}\right)}{\left\{f^{\prime \prime}\left(x_{n}\right)+f^{\prime \prime}\left(x_{n+1}^{N}\right) \pm 2 \operatorname{sign}\left(f^{\prime \prime}\left(x_{0}\right)\right) \sqrt{f^{\prime \prime}\left(x_{n}\right) f^{\prime \prime}\left(x_{n+1}^{N}\right)}\right\}}
$$

5) For $\alpha=2$ (root mean square), Formula (23) corresponds to a new cubically convergent root mean square Newton's method

$$
\begin{gathered}
x_{n+1}=x_{n}-\frac{f^{\prime}\left(x_{n}\right)}{\operatorname{sign}\left\{f^{\prime \prime}\left(x_{0}\right)\right\} \sqrt{\left\{\frac{f^{\prime \prime 2}\left(x_{n}\right)+f^{\prime \prime 2}\left(x_{n+1}^{N}\right)}{2}\right\}}(28)} \begin{array}{l}
\begin{array}{l}
\text { (21) respectively. } \\
\text { 6) New cubically convergen } \\
\text { on heronian mean is }
\end{array} \\
x_{n+1}=x_{n}-\frac{f^{\prime}\left(x_{n}\right)}{\left\{f^{\prime \prime}\left(x_{n}\right)+f^{\prime \prime}\left(x_{n+1}^{N}\right) \pm \operatorname{sign}\left(f^{\prime \prime}\left(x_{0}\right)\right) \sqrt{f^{\prime \prime}\left(x_{n}\right) f^{\prime \prime}\left(x_{n+1}^{N}\right)}\right\}}
\end{array}
\end{gathered}
$$

Some other new third-order iterative methods based on heronian mean, contra-harmonic mean, centrodial mean, logarithmic mean etc. can also be obtained from Formula

6) New cubically convergent iteration method based
7) New cubically convergent iteration method based on contra-harmonic mean is

$$
x_{n+1}=x_{n}-\frac{3 f^{\prime}\left(x_{n}\right)\left\{f^{\prime \prime}\left(x_{n}\right)+f^{\prime \prime}\left(x_{n+1}^{N}\right)\right\}}{\left\{f^{\prime \prime 2}\left(x_{n}\right)+f^{\prime \prime 2}\left(x^{N}{ }_{n+1}\right)\right\}}
$$

8) New cubically convergent iteration method based on centroidal mean is

$$
x_{n+1}=x_{n}-\frac{3 f^{\prime}\left(x_{n}\right)\left\{f^{\prime \prime}\left(x_{n}\right)+f^{\prime \prime}\left(x_{n+1}^{N}\right)\right\}}{2\left\{f^{\prime \prime 2}\left(x_{n}\right)+f^{\prime \prime 2}\left(x_{n+1}^{N}\right)+f^{\prime \prime}\left(x_{n}\right) f^{\prime \prime}\left(x_{n+1}^{N}\right)\right\}}
$$

9) New cubically convergent iteration method based on logarithmic mean is

$$
x_{n+1}=x_{n}-\frac{f^{\prime}\left(x_{n}\right)\left\{\log \left|f^{\prime \prime}\left(x_{n}\right)\right|-\log \left|f^{\prime \prime}\left(x_{n+1}^{N}\right)\right|\right\}}{f^{\prime \prime}\left(x_{n}\right)-f^{\prime \prime}\left(x_{n+1}^{N}\right)}
$$

\section{Convergence Analysis}

Theorem 5.1 Let $\beta \in I$ be an optimum point of a sufficiently differentiable function $f(x): I \subseteq \mathfrak{R} \rightarrow \mathfrak{R}$ for an open interval $I$. If the initial guess $x_{0}$ is suffi- 
ciently close to $\beta$, then for $\alpha \in \mathfrak{R}$, the family of methods defined by (23) has cubic convergence with the following error equation

$$
e_{n+1}=\left\{2 c_{4}+\frac{9}{2} c_{3}(1-\alpha)\right\} e_{n}^{3}+O\left(e_{n}^{4}\right)
$$

where $x_{n}=\beta+e_{n}$ and $c_{k}=\frac{1}{k !} \frac{f^{k}(\beta)}{f^{\prime \prime}(\beta)}, k=3,4, \ldots$.

Proof: Let $\beta$ be an extremum of the function $f(x)$ (i.e. $f^{\prime}(\beta)=0$ and $f^{\prime \prime}(\beta) \neq 0 \quad$ ). Since $f(x)$ is sufficiently differentiable function, expanding $f\left(x_{n}\right)$, $f^{\prime}\left(x_{n}\right)$ and $f^{\prime \prime}\left(x_{n}\right)$ about $x=\beta$ by means of Taylor's expansion, we have

$$
\begin{gathered}
f\left(x_{n}\right)=f(\beta)+\frac{1}{2 !} f^{\prime \prime}(\beta) e_{n}^{2}+\frac{1}{3 !} f^{\prime \prime \prime}(\beta) e_{n}^{3}+O\left(e_{n}^{4}\right) \\
f^{\prime}\left(x_{n}\right)=f^{\prime \prime}(\beta)\left[e_{n}+3 c_{3} e_{n}^{2}+4 c_{4} e_{n}^{3}+O\left(e_{n}^{4}\right)\right] \\
f^{\prime \prime}\left(x_{n}\right)=f^{\prime \prime}(\beta)\left[1+6 c_{3} e_{n}+12 c_{4} e_{n}^{2}+O\left(e_{n}^{3}\right)\right]
\end{gathered}
$$

Using (35) and (36) in (19), we get

$$
\begin{aligned}
f^{\prime \prime}\left(x_{n+1}^{N}\right)= & f^{\prime \prime}(\beta)\left[1+18 c_{3}^{2} e_{n}^{2}\right. \\
& \left.+6 c_{3}\left(8 c_{4}-18 c_{3}^{2}\right) e_{n}^{3}+O\left(e_{n}^{4}\right)\right]
\end{aligned}
$$

Case 1) For $\alpha \in \mathfrak{R} \backslash\{0\}$, Formula (23) may be written as

$$
x_{n+1}=x_{n}-\frac{f^{\prime}\left(x_{n}\right)}{f^{\prime \prime}\left(x_{n}\right)}\left[\frac{1+\left\{\frac{f^{\prime \prime}\left(x_{n+1}^{N}\right)}{f^{\prime \prime}\left(x_{n}\right)}\right\}^{\alpha}}{2}\right]^{-\frac{1}{\alpha}}
$$

Using binomial theorem and the Formulae (35), (36) and (37) in (38), we finally obtain

$$
e_{n+1}=\left\{\frac{9}{2} c_{3}(1+\alpha)+2 c_{4}\right\} e_{n}^{3}+O\left(e_{n}^{4}\right)
$$

Case 2) For $\alpha \rightarrow 0$, Formula (23) can be written as

$$
x_{n+1}=x_{n}-\frac{f^{\prime}\left(x_{n}\right)}{\operatorname{sign}\left\{f^{\prime \prime}\left(x_{0}\right)\right\} \sqrt{\left\{f^{\prime \prime}\left(x_{n}\right) f^{\prime \prime}\left(x_{n+1}^{N}\right)\right\}}}
$$

Upon using (35), (36) and (37), we obtain

$$
\begin{aligned}
& \frac{f^{\prime}\left(x_{n}\right)}{\operatorname{sign}\left\{f^{\prime \prime}\left(x_{0}\right)\right\} \sqrt{\left\{f^{\prime \prime}\left(x_{n}\right) f^{\prime \prime}\left(x_{n+1}^{N}\right)\right\}}} \\
& =e_{n}-\left(\frac{9}{2} c_{3}{ }^{2}+2 c_{4}\right) e_{n}^{3}+O\left(e_{n}^{4}\right)
\end{aligned}
$$

From (40) and (41), we obtain

$$
e_{n+1}=\left(4.5 c_{3}^{2}+2 c_{4}\right) e_{n}^{3}+O\left(e_{n}^{4}\right)
$$

Therefore, it can be concluded that for all $\alpha \in \mathfrak{R}$, the $\alpha$ th-power mean iterative family (23) for unconstrained optimization problems converges cubically. On similar lines, we can prove the convergence of the remaining Formulae (29)-(32) respectively.

\section{Further Modifications of the Family (23)}

The two main practical deficiencies of Newton's method, the need for analytic derivatives and the possible failure to converge to the solutions from poor starting points are the key issues in unconstrained optimization problems. Family (23) and other methods (29)-(32) are also variants of Newton's method and will fail miserably if at any stage of computation, the second order derivative of the function is either zero or very close to zero. The defect of Newton's method can easily be eliminated by the simple modification of iteration process. Applying the Newton's method (19) to a modified function:

$$
f^{\prime}(x)=e^{p\left(x-x_{n}\right)} f^{\prime}(x)
$$

where $p \in \mathfrak{R}$. This function has better behavior as well as the same optimum point as $f^{\prime}(x)$; we shall get the modified Newton's method given by

$$
x_{n+1}=x_{n}-\frac{f^{\prime}\left(x_{n}\right)}{f^{\prime \prime}\left(x_{n}\right)+p f^{\prime}\left(x_{n}\right)}
$$

This is a one parameter family of Newton's iteration method for unconstrained optimization problems and do not fail if $f^{\prime \prime}\left(x_{n}\right)=0$ like Newton's method. In order to obtain the quadratic convergence, the sign of entity $p$ should be chosen so that denominator is largest in magnitude. On similar lines, we can also modify some of the above-mentioned cubically convergent variants of Newton's methods for unconstrained optimization problems. Kahya [5] has also derived similar formula by using the different approach based on the ideas of Mamta et al. [16]. Similar approaches for finding the simple root of a nonlinear equation or system of equations have been used by Ben-Israel [17] and, Kanwar and Tomar [18].

\section{Numerical Results}

In this section, we shall present the numerical results 
Table 1. Test problems

\begin{tabular}{cccc}
\hline No. & Examples & Initial guess & Optimum point $(\beta)$ \\
\hline 1 & $x^{4}-8.5 x^{3}-31.0625 x^{2}-7.59 x+45$ & $\begin{array}{c}7.0 \\
10.0\end{array}$ & 8.278462409973145 \\
\hline 2 & $e^{x}-3 x^{2}$ & -1.0 & 0.204481452703476 \\
\hline 3 & $\cos x+(x-2)^{2}$ & 1.0 & 2.35424280166260 \\
\hline \multirow{2}{*}{4} & $\frac{10.2}{x}+6.2 x^{3}, x>0$ & 0.5 & 0.860054146289254 \\
\hline \multirow{2}{*}{5} & $\frac{3774.522}{x}+2.27 x-181.529, x>0$ & 32.0 & 40.777259826660156 \\
\hline
\end{tabular}

Table 2. Comparison table

\begin{tabular}{|c|c|c|c|c|c|c|c|c|c|c|}
\hline Examples & $N M$ & $A M N$ & $H M N$ & $G M N$ & $\begin{array}{c}\text { Method } \\
\text { (27) }\end{array}$ & $R M S N M$ & HERNM & $C N M$ & $T M N M$ & $L M N M$ \\
\hline \multirow[t]{2}{*}{1} & 5 & 4 & 3 & 5 & 4 & 4 & 4 & 4 & 4 & 3 \\
\hline & 5 & 4 & 3 & 4 & 4 & 4 & 4 & 4 & 4 & 3 \\
\hline \multirow[t]{2}{*}{2} & 5 & 3 & 4 & 5 & 3 & 4 & 3 & 4 & 3 & 3 \\
\hline & 5 & 3 & 4 & 5 & 4 & 4 & 4 & 3 & 3 & 2 \\
\hline \multirow[t]{2}{*}{3} & 5 & 4 & 4 & 5 & 4 & 4 & 4 & 4 & 4 & 2 \\
\hline & 4 & 3 & 3 & 4 & 3 & 3 & 3 & 3 & 3 & 2 \\
\hline \multirow[t]{2}{*}{4} & 6 & 4 & 4 & 6 & 4 & 4 & 4 & 5 & 4 & 3 \\
\hline & 5 & 4 & 4 & 5 & 4 & 4 & 4 & 4 & 4 & 3 \\
\hline \multirow[t]{2}{*}{5} & 5 & 4 & 4 & 5 & 4 & 4 & 4 & 4 & 4 & 3 \\
\hline & 5 & 4 & 3 & 5 & 4 & 4 & 4 & 4 & 4 & 3 \\
\hline
\end{tabular}

obtained by employing the iterative methods namely Newton's method ( $N M$ ), arithmetic mean Newton's method ( $A M N$ ), harmonic mean Newton's method ( $H M N)$, geometric mean Newton's method ( GMN), method (27), root mean square Newton's method ( $R M S N M$ ), heronian mean Newton's method ( HENM ), contra-harmonic mean Newton's method ( $C M N$ ), centroidal mean Newton's method ( TMN ), logarithmic mean Newton's method (LMNM) respectively to compute the extrememum of the function given in Table 1. We use the same functions as Kahya $[6,7]$. The results are summarized in Table 2. We use $\in=10^{-15}$ as tolerance. Computations have been performed using $C^{++}$in double precision arithmetic. The following stopping criteria are used for computer programs:

$$
\text { 1) }\left|x_{n+1}-x_{n}\right|<\epsilon, \quad \text { 2) }\left|f^{\prime}\left(x_{n+1}\right)\right|<\epsilon .
$$

\section{Conclusions}

It is well-known that the quadrature formulas play an important and significant role in the evaluations of definite integrals. We have shown that these quadrature formulas can also be used to develop some iterative methods for solving unconstrained optimization problems. Further, this work proposes a family of Newton-type methods based on nonlinear means and can be used to solve efficiently the unconstrained optimization problems. Proposed family (23) unifies some of the most known third-order methods for unconstrained optimization problems. It also provides many more unknown processes. All of the proposed third-order methods require three function evaluations per iterations so that their efficiency index is 1.44 , which is better than the one of Newton's method 1.41. Finally experimental results showed that the harmonic mean and logarithmic mean Newton's method are the best among the proposed iteration methods.

\section{Acknowledgement}

We would like to thank the anonymous referee for his or her valuable comments on improving the original manuscript. We are also thankful to Professor S.K. Tomar, Department of Mathematics, Panjab University, Chandigarh for several useful suggestions. Ramandeep Behl further acknowledges the financial support of CSIR, New Delhi.

\section{References}

[1] B. T. Ployak, “Newton's method and its use in optimiza- 
tion," European Journal of Operation Research, Vol. 181, pp. 1086-1096, 2007.

[2] Y. P. Laptin, "An approach to the solution of nonlinear unconstrained optimization problems (brief communications)," Cybernetics and System Analysis, Vol. 45, No. 3, pp. 497-502, 2009.

[3] G. Gundersen \& T. Steihaug, "On large-scale unconstrained optimization problems and higher order methods," Optimization methods \& Software, DOI: 10.1080/ 10556780903239071 , No. 1-22, 2009.

[4] H. B. Zhang, "On the Halley class of methods for unconstrained optimization problems," Optimization Methods \& Software, DOI: 10.1080/10556780902951643, No. 1-10, 2009.

[5] E. Kahya, "Modified secant-type methods for unconstrained optimization," Applied Mathematics and Computation, Vol. 181, No. 2, pp. 1349-1356, 2007.

[6] E. Kahya \& J. Chen, "A modified Secant method for unconstrained optimization," Applied Mathematics and Computation, Vol. 186, No. 2, pp. 1000-1004, 2007.

[7] E. Kahya, "A class of exponential quadratically convergent iterative formulae for unconstrained optimization," Applied Mathematics and Computation, Vol. 186, pp. 1010-1017, 2007.

[8] C. L. Tseng, "A newton-type univariate optimization algorithm for locating nearest extremum," European Journal of Operation Research, Vol. 105, pp. 236-246, 1998.

[9] E. Kahya, "A new unidimensional search method for optimization: Linear interpolation method," Applied Mathematics and Computation, Vol. 171, No. 2, pp. 912-
926, 2005.

[10] M. V. C. Rao \& N. D. Bhat, "A new unidimensional search scheme for optimization," Computer \& Chemical Engineering, Vol. 15, No. 9, pp. 671-674, 1991.

[11] P. S. Bulle, "The power means, hand book of means and their inequalities," Kluwer Dordrecht, Netherlands. 2003.

[12] J. E. Dennis \& R. B. Schnable, "Numerical methods for unconstrained optimization and nonlinear equations," Prentice-Hall, New York, 1983.

[13] S. Weerakoon \& T. G. I. Fernando, "A variant of Newton's method with accelerated third-order convergence," Applied Mathematics Letter, Vol. 13, pp. 87-93, 2000.

[14] M. Frontini \& E. Sormani," Some variants of Newton's method with third-order convergence," Applied Mathematics and Computation, Vol. 140, pp. 419-426, 2003.

[15] W. Gautschi, "Numerical analysis: an introduction," Birkhäuser, Boston, Inc., Boston, 1997.

[16] Mamta, V. Kanwar, V. K. Kukreja \& S. Singh, "On a class of quadratically convergent iteration formulae," Applied Mathematics Computation, Vol. 166, pp. 633-637, 2005.

[17] B. I. Adi, "Newton's method with modified functions," Contemporary Mathematics, Vol. 204, pp. 39-50, 1997.

[18] V. Kanwar \& S. K. Tomar, "Exponentially fitted variants of Newton's method with quadratic and cubic convergence," International Journal of Computer Mathematics, Vol. 86, No. 9, pp. 603-611, 2009. 